\title{
Neuroenteric cysts of the brain-comprehensive magnetic resonance imaging
}

\author{
Divyata R Hingwala, Neelima Radhakrishnan¹, Chandrasekharan Kesavadas, Bejoy Thomas, \\ Tirur Raman Kapilamoorthy, Vishnupuri V Radhakrishnan¹ \\ Departments of Imaging Sciences and Interventional Radiology, and 'Pathology, Sree Chitra Tirunal Institute for Medical Sciences \\ and Technology, Thiruvananthapuram, Kerala, India
}

Correpondence: Dr. Divyata R Hingwala, Department of Imaging Sciences and Interventional Radiology, Sree Chitra Tirunal Institute for Medical Sciences and Technology, Thiruvananthapuram - 695 011, Kerala, India. E-mail: divyatahingwala@gmail.com

\section{Abstract}

Neuroenteric cysts are developmental cysts that should be differentiated from other, more common non-neoplastic cysts as well as cystic neoplasms. While these lesions may have varied imaging findings, T1 hyperintense prepontine lesion due to mucinous/ proteinaceous content is characteristic. Location and imaging characteristics aid in formulating the correct diagnosis of neuroepithelial/ neuroenteric cysts. Magnetic resonance spectroscopy (MRS) has the specific finding of N-Acetyl Aspartate (NAA)-like peak at $2.02 \mathrm{ppm}$ which is not seen in other cystic lesions. In this study, we aim to discuss the imaging findings of these lesions on conventional and advanced MRI findings and provide radiologic-pathologic correlation. We also briefly describe the pathogenesis, embryology and radiological differential diagnoses of these cysts.

Key words: Diffusion tensor imaging; magnetic resonance imaging; magnetic resonance spectroscopy; neuroenteric cyst; neuroepithelial cyst

\section{Introduction}

Neuroenteric cysts of the brain are uncommon developmental cysts that should be differentiated from other, more common non-neoplastic cysts as well as cystic neoplasms. The pathogenesis of these cysts is poorly understood. Based on the development and the proposed cell of origin, the epithelial cysts have been classified as neuroepithelial and neuroenteric cysts. ${ }^{[1]}$

In this case series, we aim to discuss the varied imaging findings of neuroenteric cysts including conventional and advanced MRI findings and provide radiologic-pathologic

\begin{tabular}{|l|l|}
\hline \multicolumn{2}{|c|}{ Access this article online } \\
\hline Quick Response Code: & \\
\hline & Website: \\
\hline & www.ijriorg \\
\cline { 2 - 3 } & DOI: \\
\hline & $10.4103 / 0971-3026.116579$ \\
\hline
\end{tabular}

correlation. We also briefly describe the pathogenesis, embryology and radiological differential diagnoses of these cysts.

\section{Materials and Methods}

\section{Case selection}

We performed a retrospective analysis of five surgically proven cases of neuroenteric cysts of the brain. The patients ranged in age from 10 to 32 years with a mean age of 20.8 years. All the patients were females.

\section{Imaging technique}

Three of the five patients were evaluated with computed tomography (CT). All patients were evaluated with MRI performed on a $1.5 \mathrm{~T}$ clinical MR scanner (Magnetom Avanto SQ Engine, Seimens, Erlangen, Germany). Contrast-enhanced scans were available in three patients. Fluid-attenuated inversion recovery (FLAIR) sequence was performed in two patients. Diffusion imaging was performed in all patients. Diffusion tensor imaging (DTI) and susceptibility-weighted imaging (SWI) were available 
in two patients. $\mathrm{T}^{*}$ gradient recalled echo (GRE) was available in two patients. MR spectroscopy was available in three patients.

\section{Results}

\section{Clinical presentation}

Patient history was available from medical records. The commonest presenting complaint was headache and neck pain. Other symptoms included quadriparesis, gait unsteadiness, downgaze preference, and somnolence in one patient each.

\section{Location, size and morphology}

All the cysts were extra-axial. Four of five cysts were located infratentorially [prepontine (one), cerebello pontine angle (one), foramen magnum (one), fourth ventricle (one)]. All of these extended to the midline. One was located in the midline in the suprasellar cistern extending into the posterior fossa in the interpeduncular and prepontine cisterns.

All the cysts were oblong in shape, well-defined and had smooth, regular margins. Two of the cysts were multilobulated with smooth, scalloped margins. The dimensions ranged from 1.9 to $5.9 \mathrm{~cm}$.

\section{Imaging findings}

CT scan was available in three patients. The attenuation varied on non-enhanced CT. As compared to brain parenchyma, one cyst was hypodense, one was isodense and one was hyperdense. A hyperattenuating focus of calcification was present in the hyperdense cyst. No obvious bony defects were seen.

On MRI, the cysts had signal intensities varying from cerebrospinal fluid (CSF)-like signal in T1 and T2-weighted images to T1 hyperintense or T2 hypointense signal. On T1-weighted sequences, three of the cysts were hyperintense and two were hypointense as compared with CSF signal. One of the three $\mathrm{T} 1$ hyperintense cysts had undergone frequency-selective fat saturation imaging. It did not show loss of signal of the entire content, thus lipid content was excluded. Four cysts were hyperintense on T2-weighted images and one cyst was hypointense. FLAIR sequence was available in two cases. One cyst was hyperintense on FLAIR and signal was completely suppressed in one case (hypointense). There was no enhancement with gadolinium-enhanced scans.

The diffusion in the cyst contents was facilitated. The apparent diffusion coefficient (ADC) values (available in four patients) ranged from $0.281 \times 10^{-3} \mathrm{~mm}^{2} / \mathrm{s}$ to $2.25 \times 10^{-3} \mathrm{~mm}^{2} / \mathrm{s}$ with a mean ADC value of $1.35 \times 10^{-3} \mathrm{~mm}^{2} / \mathrm{s}$.

Susceptibility-weighted imaging (available in two patients) showed focus of blooming in one patient which corresponded with the hyperattenuating calcific focus. No blooming was visualized in the two cases with GRE images.

Three of our patients had undergone MR. All patients showed a peak at $2.02 \mathrm{ppm}$ corresponding to NAA-like compounds. Spectrum from a voxel placed in the cyst showed a mean NAA/Cr ratio of 10.2 and a mean $\mathrm{Cho/Cr}$ ratio of 0.77 . One of the patients had spectra sampled from normal reference voxels. It showed a NAA/Cr ratio of 2.27 and a mean Cho/Cr ratio of 1.28 .

Pre-operative diagnosis neuroepithelial/neuroenteric cyst was made in three cases and arachnoid cyst was made in two cases.

The imaging findings for all patients are summarized in Tables $1 \mathrm{~A}$ and B.

\section{Pathology}

All patients had undergone surgery and had histopathology available. Microscopic examination of the cyst wall revealed two major histopathologic patterns depending upon the presence or absence of ciliated columnar epithelium, goblet cells or squamous epithelium. Two cases had cuboidal or ciliated columnar epithelium with goblet cells or mucous glands. One case had ciliated columnar epithelium, mucosal glands and stratified squamous epithelium. One case with ciliated epithelium and squamous metaplasia had a fibrohyaline nodule with cholesterol cleft but no goblet cells. All these were classified as neurenteric cysts. Two cases had pseudostratified ciliated columnar to cuboidal epithelium without goblet cells. These were classified as neuroepithelial cysts on microscopic examination.

Immunohistochemical staining with Periodic acid Schiff

Table 1A: Imaging findings on conventional MRI

\begin{tabular}{|c|c|c|c|c|c|c|c|c|}
\hline Patient code & CT & T1 & $\mathrm{T} 2$ & FLAIR & DWI & SWI & $\mathrm{Gd}+$ & Preoperative diagnosis \\
\hline 1 & Iso & Hyper & Hyper & NA & Facilitated & NA & NA & $\mathrm{NE}$ \\
\hline 2 & Hyper, focal calcification & Hyper & Нуро & NA & Facilitated & 1 nodule & 0 & NE \\
\hline 3 & NA & Нуро & Hyper & NA & Facilitated & NA & NA & Arachnoid cyst \\
\hline 4 & NA & Hyper & Hyper & Hyper & Facilitated & GRE-0 & 0 & $\mathrm{NE}$ \\
\hline 5 & Нуро & Нуро & Hyper & Нуро & Facilitated & GRE-0 & 0 & Arachnoid cyst \\
\hline
\end{tabular}


Table 1B: Imaging findings on DTI and IMR spectroscopy ( $\mathrm{TE}=135 \mathrm{msec}$ )

\begin{tabular}{lcc}
\hline Patient code & MIRS NAA/Cr & MRS Cho/Cr \\
\hline 1 & 17.63 & 0.225 \\
2 & 6.467 & 1.33 \\
3 & NA & NA \\
4 & 6.512 & 0.74 \\
5 & NA & NA \\
\hline
\end{tabular}

NA: Not available, ADC: Apparent diffusion coefficient, ADC SD: Standard deviation of ADC, FA mean: Mean fractional anisotropy, FA SD: Standard deviation of FA, NAA/Cr:

$\mathrm{N}$ acetyl aspartate like compound to Creatinine ratio, Cho/Cr: Choline to creatinine ratio, MRS: Magnetic resonance spectroscopy, DTI: Diffusion tensor imaging

stain (PAS) and Glial fibrillary acidic protein (GFAP) was done in all cases which were GFAP negative and did not show PAS positive basement membrane suggestive of neuroenteric cysts. Immunohistochemical staining was not suggestive of neuroepithelial cyst in any case. Hence, the final pathologic diagnosis was neuroenteric cyst in all cases. The pathology findings are described in Table 2.

\section{Imaging-pathology correlation}

The cases with detailed histopathology reports were subdivided into three groups. Cysts with cuboidal or ciliated epithelium and mucin-secreting goblet cells were hyperintense on $\mathrm{T} 1$ and T2-weighted images (two cases). One of these cases also had squamous epithelium. One of these cases had FLAIR images available which showed hyperintense cyst content. Mean ADC values of neuroenteric cysts were $1.699 \times 10^{-3} \mathrm{~mm}^{2} / \mathrm{s}$. The cyst with squamous metaplasia but no goblet cells was hyperintense on T1 and hypointense on T2-weighted images. The cysts with pseudostratified ciliated columnar to cuboidal epithelium without goblet cells were hypointense on T1 and hyperintense on T2-weighted images. FLAIR images available in one patient had hypointense signal; however, it was not completely suppressed like CSF signal.

Representative cases highlighting the imaging findings are described below and illustrated in Figures 1-5.

\section{Case Reports}

\section{Case 1}

A 17-year-old female patient presented with neck pain since two weeks which was aggravated by exercise and neck movements. She developed weakness of limbs for one week which started in left upper limb and then evolved to involve all four limbs. She also complained of numbness in both upper limbs and urgency of micturation. On neurological examination, tone was mildly decreased in both upper limbs and increased in both lower limbs. Power was 2/5 in left upper limbs and 4/5 in other limbs. Deep tendon reflexes were absent in upper limbs and exaggerated in lower limbs. Pain and touch sensation decreased by $30 \%$ below C2 level and vibration sense lost below T2 level.
Table 2: Histopathology

\begin{tabular}{ll}
\hline Patient code & Histopathology description \\
\hline 1 & $\begin{array}{l}\text { Cuboidal columnar epithelium, goblet cells } \\
2\end{array}$ \\
3 & $\begin{array}{l}\text { Pseudostratified ciliated columnar epithelium with squamous } \\
\text { metaplasia, fibrohyaline nodule with cholesterol cleft }\end{array}$ \\
4 & $\begin{array}{l}\text { Ciliated pseudostratified cuboidal to flattened epithelium } \\
\text { Ciliated columnar, pseudostratified columnar, stratified } \\
\text { squamous, mucosal glands, clusters of Rosenthal fibers }\end{array}$ \\
5 & Cuboidal or columnar epithelium (pseudostratified at some foci)
\end{tabular}

MRI [Figure 1] showed a well circumscribed extra-axial cystic lesion of $3 \times 1.8 \times 2.4 \mathrm{~cm}$ size noted anterior and to the right of medulla extending through foramen magnum into upper cervical canal. It was hyperintense on T1 and T2 weighted images with no diffusion restriction. On right side hypoglossal nerve was displaced laterally by lesion.

She underwent suboccipital craniectomy and total excision of the lesion via right lateral approach. Histopathology showed a cyst wall is lined by cuboidal and columnar epithelium with goblet cells (arrow) suggestive of a neuroenteric cyst.

Postoperatively, patient's power improved to $4 / 5$ in all 4 limbs.

Case 2

A 28-year-old lady presented with headache and vomiting since $11 / 2$ years. She complained of difficulty in gripping objects with left upper limb, gait unsteadiness with tendency to sway to the left side, dysarthria and nasal regurgitation on swallowing fluids. On examination, bilateral gaze evoked downbeat nystagmus was present. Gag reflex was sluggish bilaterally but uvula was central. There was drooping of right shoulder and weakness of right sternocleidomastoid. Left cerebellar signs were present with ataxic gait. Bulk and tone of muscles was normal.

CT [Figure 2] showed a hyperdense extraxial lesion in left side posterior fossa, compressing brainstem and distorting the $4^{\text {th }}$ ventricle with obstructive hydrocephalus. On MRI [Figure 2], it appeared iso- to mildly hypointense on T2 weighted images and hyperintense on T1 weighted images without suppression of signal on fat saturated images. The lesion measured $5.4 \times 4.2 \times 5.34 \mathrm{cms}$. There was no obvious enhancement on administration of contrast. There was no restriction diffusion. On the anterior aspect of the lesion, just to the left of midline, there was a T1- and T2 hypointense focus that corresponded with slightly increased density on plain CT and showed blooming on SWI (likely calcification). The lesion showed facilitated diffusion. MRS showed a large NAA like peak at $2.02 \mathrm{ppm}$.

She underwent midline suboccipital craniectomy and near total excision of the lesion. Histopathology showed a 

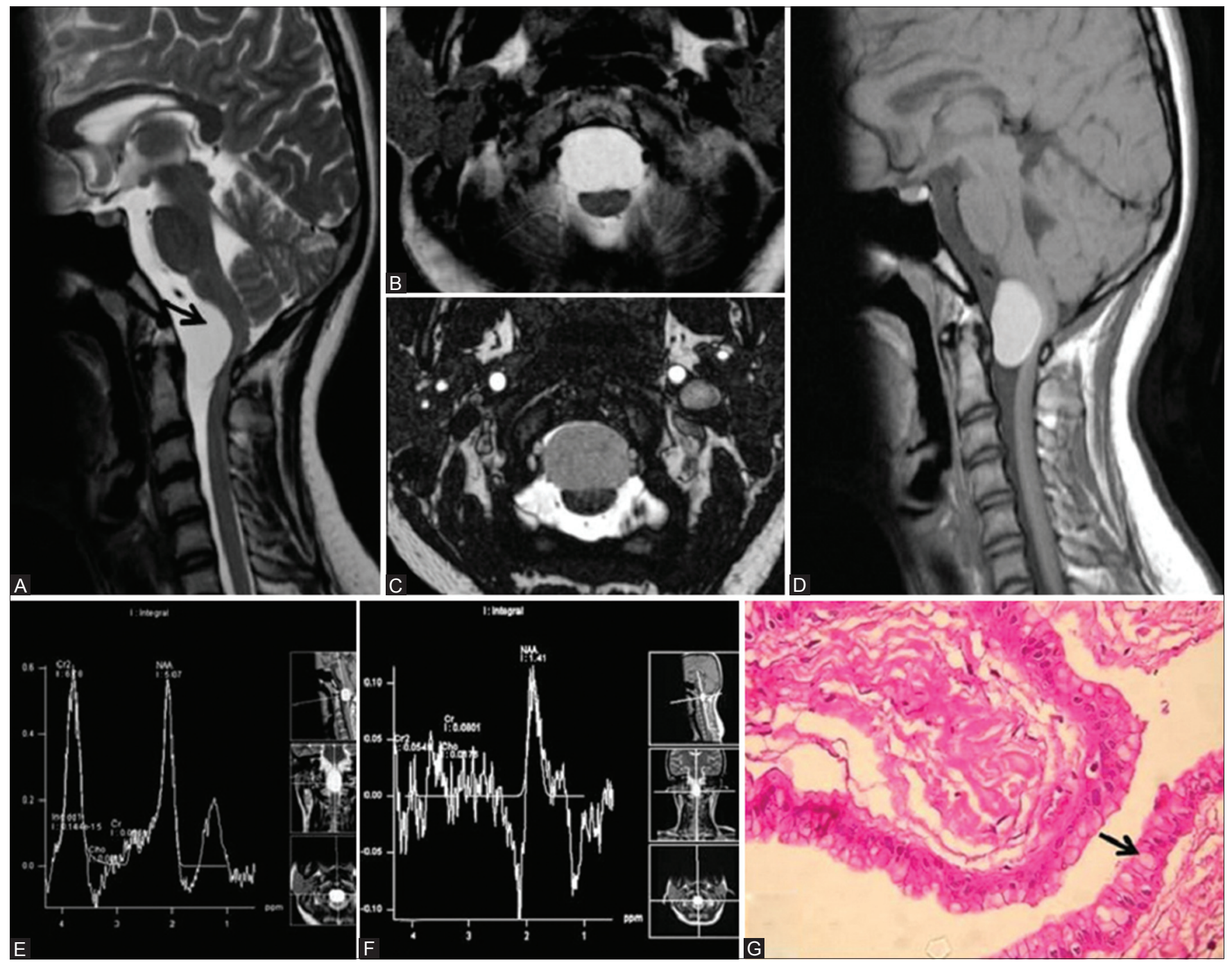

Figure 1 (A-G): T2W sagittal (A) and axial (B) images show an oblong, well-defined hyperintense cyst (arrow) anterior to the cervico-medullary junction. CISS axial image $(C)$ shows that the cyst content is hypointense and not like CSF. The cyst contents are hyperintense on T1W sagittal image (D). MRS at TE $35 \mathrm{msec}(\mathrm{E})$ and $135 \mathrm{msec}(\mathrm{F})$ show a prominent peak at $2.02 \mathrm{ppm}(\mathrm{G})$. Histopathology-cyst wall is lined by cuboidal and columnar epithelium with goblet cells (arrow)-neuroenteric cyst

cyst wall composed of pseudostratified ciliated columnar epithelium (black arrow) with extensive areas of squamous metaplasia suggestive of neuroenteric cyst.

\section{Case 3}

A 7 month old girl presented with complaints of poor feeding, excessive crying and downgaze preference since 1 month. MRI [Figure 3] showed a CSF-intensity cystic lesion of $7 \times 5.7 \times 5.8 \mathrm{~cm}$ size in left side of posterior fossa compressing the brainstem and cerebellar hemisphere to right with obstructive hydrocephalus and periventricular hyperintensities. The child underwent midline suboccipital craniotomy and decompression of cyst. On histopathology, the cyst wall was lined by pseudostratified ciliated cuboidal to flattened epithelium (black arrow) with a subepithelium composed of fibrocollagenous tissue with dilated vascular channels. On microscopic examination, the findings were suggestive of a neuroepithelial cyst, but based on the immunohistochemical properties, a final diagnosis of neuroenteric cyst was made.

\section{Case 4}

32-year-old lady presented with complaints of oligomenorrhoea since 9 years; tremor of both hands since 9 months; intermittent right frontal headache, drop attacks, urinary incontinence, somnolence and irrelevant laughing since 6 months. On examination, there was bilateral secondary optic atrophy. No other cranial nerve involvement. Power was grade $3 / 5$ in both upper limbs and grade $4+/ 5$ in both lower limbs. Deep tendon reflexes were brisk. Bilateral cerebellar signs were present.

MRI [Figure 4] showed a suprasellar lesion elevating the floor of the fourth ventricle and extending into the interpeduncular fossa posteriorly. It appeared iso- to mildly 

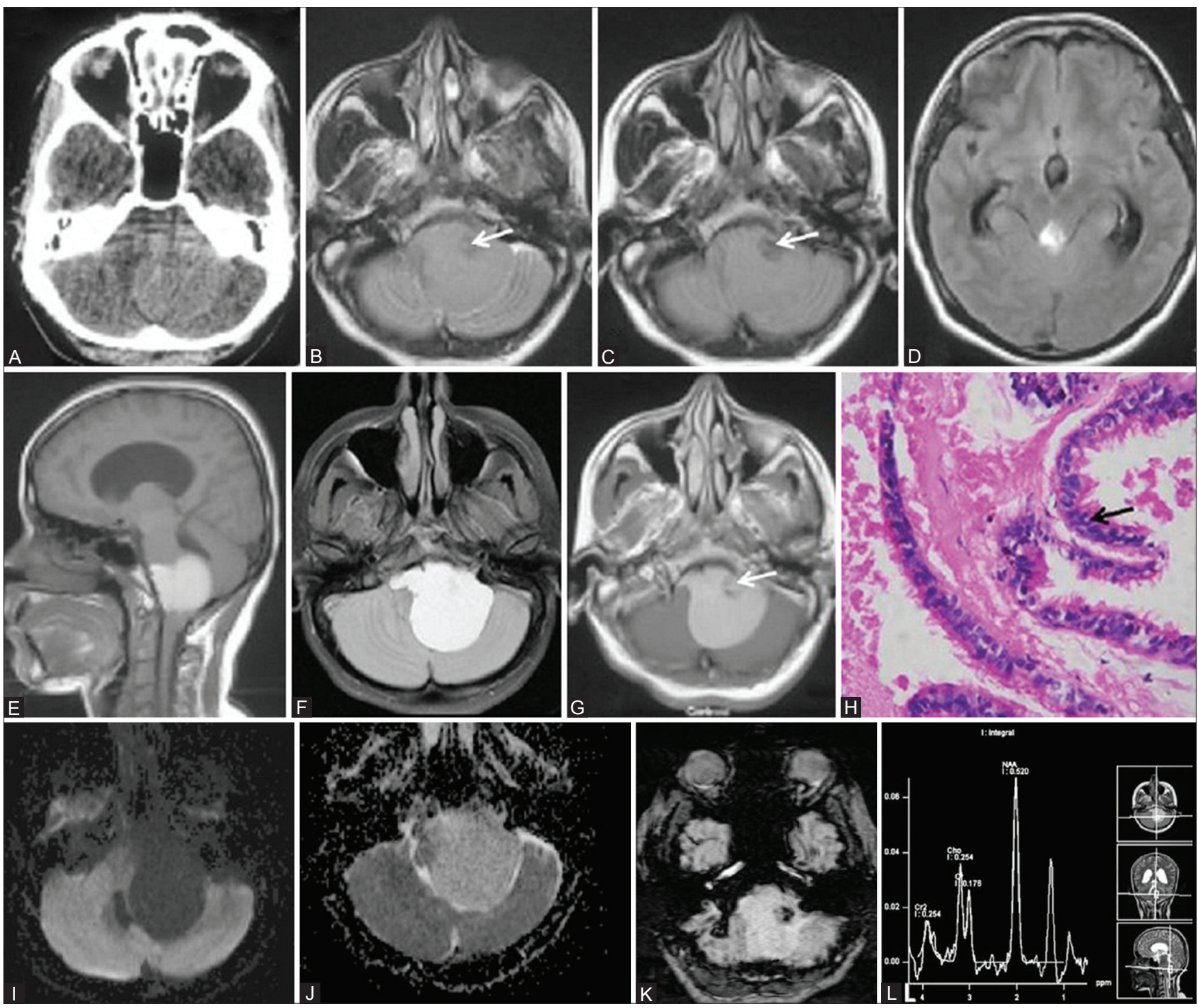

Figure 2 (A-L): Plain CT (A) shows a hyperdense lesion in the posterior fossa extending to the left cerebellopontine angle. T2W (B) and FLAIR (C,D) axial images show an extra-axial cyst with hypointense fluid and resultant obstructive hydrocephalus (D). A smaller hypointense focus is also seen (white arrow). T1W sagittal (E) and fat-saturated axial images ( $F$ ) show that the cyst content is hyperintense without loss of signal intensity on fat suppression. Post-contrast axial images $(\mathrm{G})$ do not show contrast enhancement. Histopathology-cyst wall is composed of pseudostratified ciliated columnar epithelium (black arrow) with extensive areas of squamous metaplasia-neuroenteric cyst $(\mathrm{H})$ Diffusion Trace image (I) and ADC map (J) shows facilitated diffusion. SWI image (K) shows a focus of blooming corresponding to the hypointense focus seen on conventional imaging. MRS TE $135 \mathrm{msec}(\mathrm{L})$ shows a large NAA-like peak at $2.02 \mathrm{ppm}$

hyperintense on $\mathrm{T} 1$ weighted images and hyperintense on T2 weighted images. There was facilitated diffusion in the lesion with high ADC values. There was no blooming on gradient echo images. There was no obvious enhancement on administration of contrast. On MR spectroscopy, there was a prominent peak at $2.02 \mathrm{ppm}$ corresponding to NAA like compound. The third and lateral ventricles were dilated with periventricular hyperintensities suggestive of transependymal seepage of CSF.

The patient underwent right frontotemporal craniotomy, single peice orbitozygomatic osteotomy and subtotal excision of the lesion. Histopathology showed ciliated columnar, pseudostratified columnar, stratified squamous, mucosal glands, clusters of Rosenthal fibres suggestive of neuroepithelial cyst. Based on the immunohistochemical properties, a final diagnosis of neuroenteric cyst was made.

Case 5

A 17-year-old female patient presented with occasional headache since two years increased since the last three months. On examination, bilateral papilledema was present. No other focal neurological deficits were present.

MRI [Figure 5] showed a well-defined CSF-intensity cystic lesion in the region of the fourth ventricle with obstructive hydrocephalus. There were no enhancing solid 

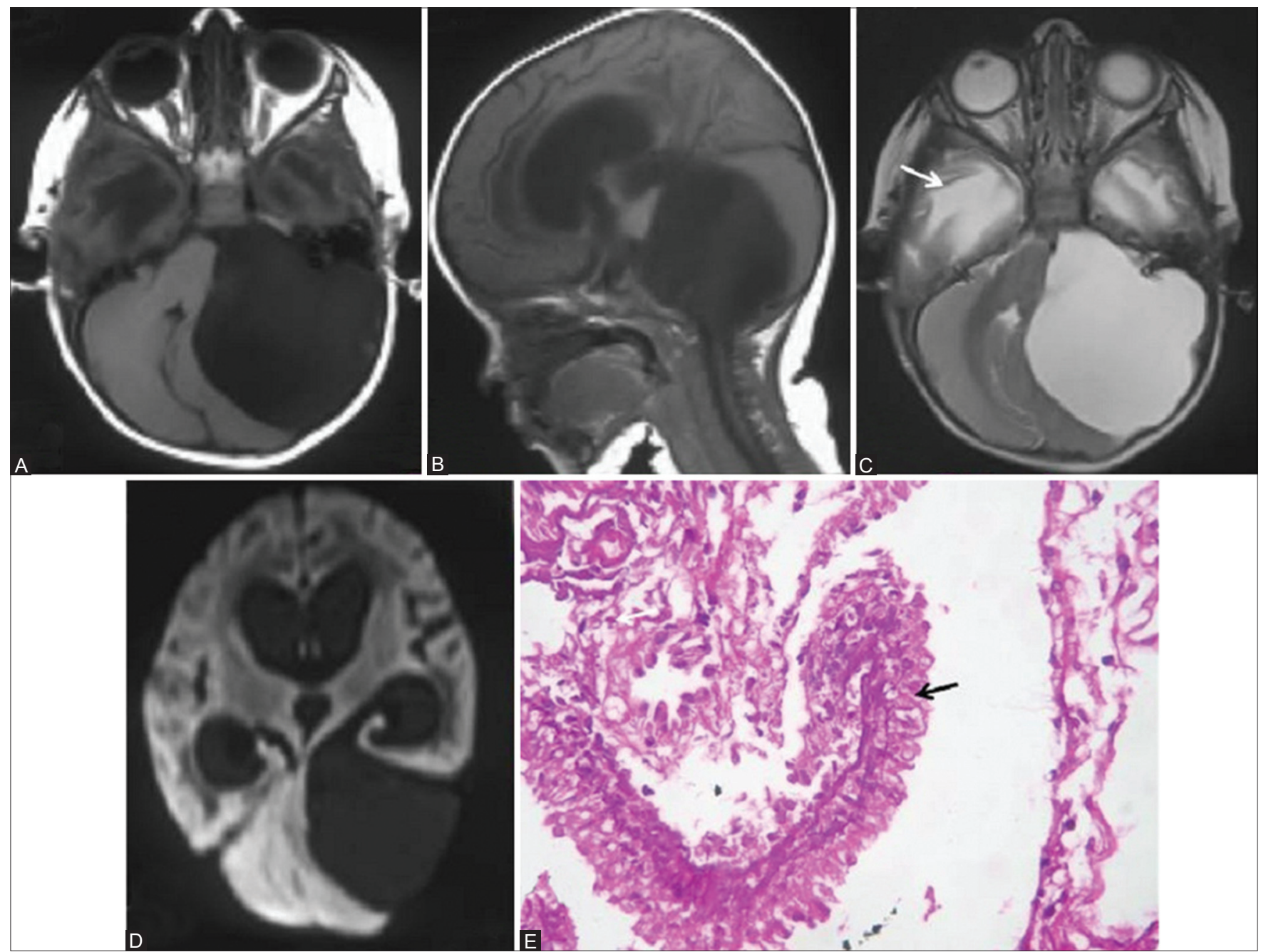

Figure $3(A-E)$ : T1W axial (A) and sagittal (B) and T2W axial (C) images show a cyst in the posterior fossa causing mass effect on the cerebellum and brainstem with obstructive hydrocephalus and periventricular seepage of CSF (white arrow). The cyst follows CSF signal intensity (hypointense on T1WI and hyperintense on T2WI) and shows facilitated diffusion (D). Histopathology-cyst wall is lined by pseudostratified ciliated cuboidal to flattened epithelium (black arrow) with a subepithelium composed of fibrocollagenous tissue with dilated vascular channels-neuroepithelial cyst (E)

components. The lesion showed facilitated diffusion. The patient underwent midline suboccipital craniotomy and deroofing of the cyst. Histopathology showed cuboidal or columnar epithelium (pseudostratified at some foci). Based on the immunohistochemical properties, a final diagnosis of neuroenteric cyst was made.Patient did well in the post-operative period with reduction of the hydrocephalus.

\section{Discussion}

Neuroenteric cysts arise around the third week of gestation due to abnormal partitioning of the embryonic notochordal plate and presumptive endoderm ${ }^{[2]}$ and persistence of the primitive neurenteric canal, notochordal anomalies or intradural adhesions between endo- and ectodermal tissues. ${ }^{[1]}$

Neuroenteric cysts are more commonly located in the spine ${ }^{[3,4]}$ and are often associated with vertebral anomalies. ${ }^{[4,5]}$ Intracranial neuroenteric cysts are reported to be less common. ${ }^{[5]}$ However, in this series, all the cases were intracranial, except one case located at the foramen magnum. None of the cases were associated with bony defects. The majority of our cases were infratentorial and located near the midline. This corresponded with the available data on intracranial neuroenteric cysts. ${ }^{[6]}$ A supratentorial off-midline frontal convexity location for neurenteric cysts has been described in case reports. ${ }^{[1,6]}$ Intraventricular (third, fourth and lateral ventricles), ${ }^{[7-9]}$ choroid plexus and intraparenchymal locations have been described for intracranial neuroepithelial cysts. One of our neuroepithelial cysts was located in the fourth ventricle while the other was located in the cerebellopontine angle.

These cysts are considered to be well-defined extra-axial lesions. On conventional MRI sequences, the signal intensity depends upon cyst contents. Neuroenteric cysts have been described to be iso- to mildly hyperintense on T1-weighted images and hyperintense on T2-weighted images due to the 

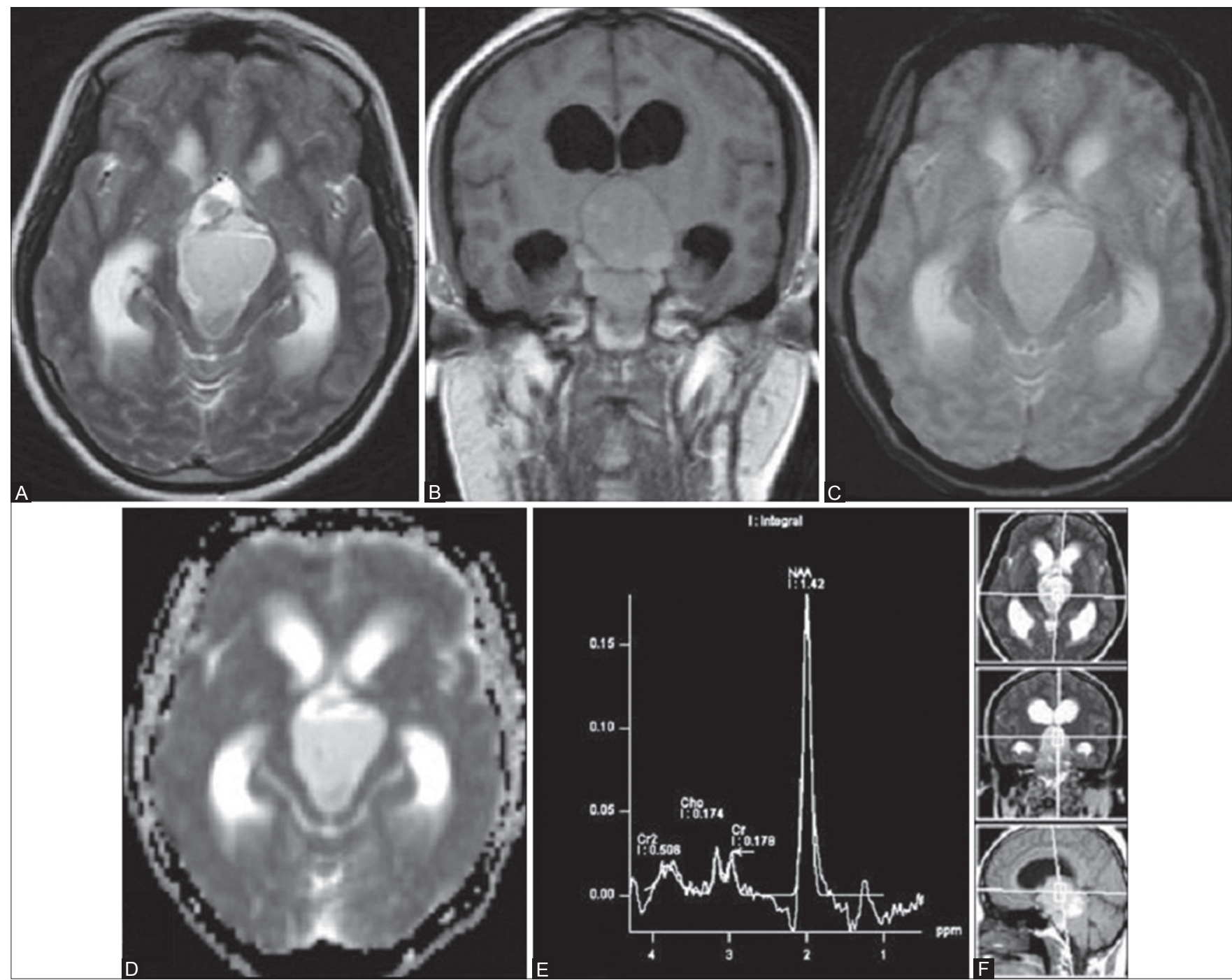

Figure 4 (A-F): MRI shows a suprasellar lesion elevating the floor of the fourth ventricle and extending into the interpeduncular fossa posteriorly It appears hyperintense on T2W axial image (A) and iso- to mildly hyperintense on T1W mid-sagittal image (B). There is no blooming on gradient echo image (C). There is facilitated diffusion in the lesion with high ADC values (D). There was no obvious enhancement on administration of contrast (E). On MR spectroscopy $(\mathrm{F})$ with a TE of $135 \mathrm{~ms}$, there is a prominent peak at $2.02 \mathrm{ppm}$ corresponding to NAA-like compound. The third and lateral ventricles are dilated with periventricular hyperintensities suggestive of transependymal seepage of CSF. (D and E with kind permission from Springer Science+Business Media: Neuroradiology (2008) 50:137-144, Periakaruppan A, et al.)

proteinaceous contents. T2 hypointense signal occurs due to inspissated contents. The typical imaging findings described are that of a small (less than $2 \mathrm{~mm}$ ) oval, multilobulated, $\mathrm{T} 1$ hyperintense prepontine or premedullary lesion. ${ }^{[5]}$ The contents of cysts are not completely suppressed on FLAIR and show mostly facilitated diffusion. Contrast enhancement and calcification are uncommon. ${ }^{[10]}$

Neuroepithelial cysts have been described to have low signal intensity on T1-weighted images and high signal intensity on T2-weighted images due to clear serous contents resembling CSF. ${ }^{[9,11,12]}$ However, T1 hyperintense and T2 hypointense neuroepithelial cyst of the fourth ventricle have been described. ${ }^{[11]}$

In this study, the cysts had variable signal intensity on MRI.
Most of the lesions were iso- hyperintense on T1-weighted and T2-weighted images. One of the neuroenteric cysts was hypointense on T2-weighted images. The neuroepithelial cysts followed CSF signal on T1-weighted and T2-weighted images but were mildly hyperintense on FLAIR. Blooming on SWI was present in one cases; one corresponded to calcification. Contrast enhancement was not seen in any of the cases.

MR spectroscopy has classical findings of a large peak at $2.02 \mathrm{ppm}$ despite the absence of neuronal elements. This corresponds to NAA-like compound in the cyst contents. ${ }^{[13-15]}$ This is secreted by the cyst lining (goblet/ciliated columnar cells). This peak helps to separate enterogenous cysts from other similar appearing cystic lesions and was initially described by Candiota et al. ${ }^{[15]}$ 

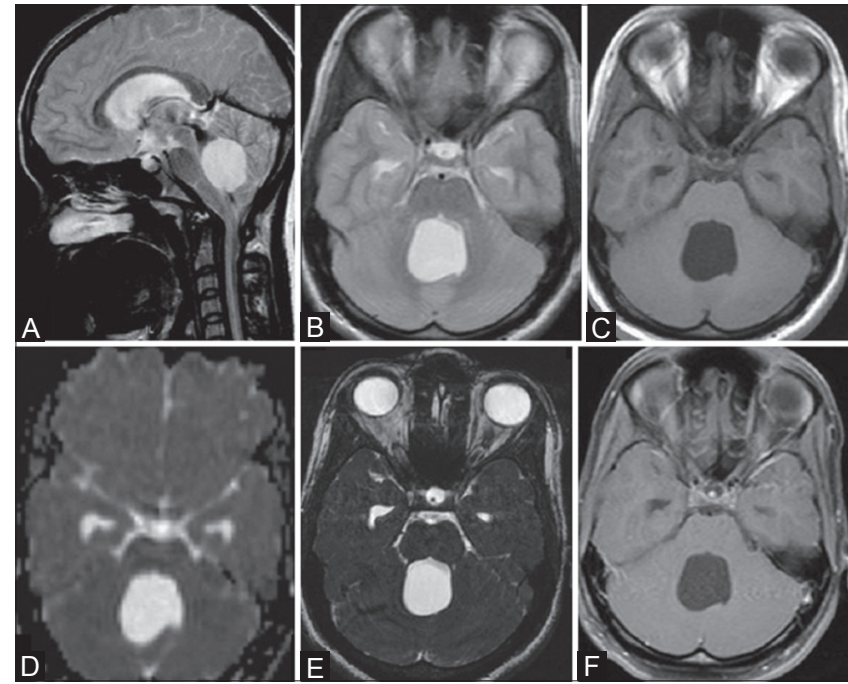

Figure 5 (A-F): MRI shows a well defined CSF-intensity cystic lesion in the region of the fourth ventricle with hyperintense signal on T2 weighted images $(A, B)$ and hypointense signal on T1 weighted image $(C)$. There is dilatation of the temporal horns of lateral ventricles (obstructive hydrocephalus). The lesion shows facilitated diffusion (D). On constructive interference at steady state (CISS) axial image (E), the cyst walls cannot be appreciated. There are no internal septations. There is no enhancement on administration of contrast (F)

Neuroenteric cysts have variable histopathological findings. Neuroenteric cysts are lined by epithelium that reflects the endodermal origin (squamous or columnar with mucin-secreting goblet cells). Based on wall structure, neuroenteric cysts are further divided into three types. ${ }^{[16]}$ Type A cysts are lined by single or pseudostratified layer of ciliated or non-ciliated cuboidal or columnar epithelium. In addition to this lining, the wall of Type B cysts also has connective tissue components, glands, smooth muscles, lymphoid tissue or nerve ganglion. The Type $C$ cysts may have glial elements like ependymal cells in the wall. All our cases corresponded to the Type A cysts. The proteinaceous-mucinous cyst content secreted by the goblet cells could account for the T1 hyperintense signal. However, Preece et al., found no correlation between the signal intensity on conventional MRI sequences and histopathology. ${ }^{[5]}$

The differentiation of neuroepithelial and neuroenteric cysts can be made on immunohistochemical staining with PAS and GFAP. PAS staining can detect the basement membrane in neuroepithelial cyst. GFAP staining is also seen in neuroepithelal cysts but not neuroenteric cyst. Intracranial neuroenteric cysts should be differentiated from other intracranial cystic lesions. CSF intensity cysts include arachnoid cysts, epidermoid cysts ${ }^{[1,6]}$ and parasitic cysts. ${ }^{[6]}$ Unlike neuroepithelial and neuroenteric cysts, arachnoid cysts have no peak at $2.02 \mathrm{ppm}$ on MR spectroscopy due to absence of neuronal elements. In addition, they suppress completely on FLAIR images and follow CSF signal intensity on all pulse sequences with facilitated diffusion.
Parasitic cysts like hydatid cyst or neurocysticercosis (NCC) can be differentiated based on the location (racemose NCC in basal cisterns) and on the presence of scolex and enhancement, depending on the stage of NCC. T1 hyperintense lesions include white epidermoids, dermoid cysts, lipomas and other endodermal cysts like Rathkes or colloid cyst. Epidermoids show restriction of diffusion and heterogeneous hyperintense signal on FLAIR images. Lipid-containing lesions like dermoids and lipomas show suppression of signal on fat-saturated images. Other cysts that must be excluded are tumoral cysts. These have variable solid components, contrast enhancement and perifocal edema. Contrast enhancement may also be seen with reactive inflammatory/infectious processes occurring in the cysts. However, none of our cases showed enhancement. MR spectroscopy in tumors shows high $\mathrm{Cho} / \mathrm{Cr}$ ratio and decreased NAA.

\section{Conclusions}

The imaging spectrum for neuroenteric cysts is broader than previously reported. Intracranial neuroenteric cysts should be considered in the differential diagnosis for intracranial extra axial cystic lesions. The signal intensity pattern varies on conventional MRI sequences. T1 hyperintense prepontine lesion due to mucinous/proteinaceous content is characteristic. Location and imaging characteristics aid in formulating the correct diagnosis of neuroepithelial/ neuroenteric cysts. The finding of NAA-like peak at $2.02 \mathrm{ppm}$ on MRS may also help to differentiate these lesions from other cysts.

\section{Acknowledgement}

We thank the Director, Sree Chitra Tirunal Institute for medical Sciences and Technology, Thiruvananthapuram for permitting us to publish this article. We also thank the Department of Pathology, Sree Chitra Tirunal Institute of Medical Sciences and Technology, Thiruvananthapuram for helping us with the histopathology.

\section{References}

1. Cheng JS, Cusick JF, Ho KC, Ulmer JL. Lateral supratentorial endodermal cyst: Case report and review of literature. Neurosurgery 2002;51:493-9.

2. Savage JJ, Casey JN, McNeill IT, Sherman JH. Neurenteric cysts of the spine. J Craniovertebr Junction Spine 2010;1:58-63.

3. Inoue T, Kawahara N, Shibahara J, Masumoto T, Usami K, Kirino T. Extradural neurenteric cyst of the cerebellopontine angle: Case report. J Neurosurg 2004;100:1091-3.

4. Gao P, Osborn AG, Smirniotopoulos JG. Neurenteric cysts: Pathology, imaging spectrum, and differential diagnosis. Int J Neuroradiol 1995;1:17.

5. Preece MT, Osborn AG, Chin SS, Smirniotopoulos JG Intracranial Neurenteric cysts: Imaging and pathology spectrum. AJNR Am J Neuroradiol 2006;27:1211-6.

6. Tan GS, Hortobagyi T, Al-Sarraj S, Connor SE. Intracranial laterally based supratentorial neurenteric cyst. Br J Radiol 2004;77: 963-5. 
7. Ormond DR, Omeis I, Mohan A, Murali R, Narayan P. Obstructive hydrocephalus due to a third ventricular neuroepithelial cyst. J Neurosurg Pediatr 2008;1:481-4.

8. Nakase H, Ishida $\mathrm{Y}$, Tada T, Sakaki T, Goda K, Tunoda S, et al. Neuroepithelial cyst of the lateral ventricle: Clinical features and treatment. Surg Neurol 1992;37:94-100.

9. Czervionke LF, Daniels DL, Meyer GA, Pojunas KW, Williams AL, Haughton VM. Neuroepithelial cysts of the lateral ventricles: MR appearance. AJNR Am J Neuroradiol 1987;8:609-13.

10. Shakudo M, Inoue Y, Ohata K, Tanaka S. Neurenteric cyst with alteration of signal intensity on follow-up MR images. AJNR Am J Neuroradiol 2001;22:496-8.

11. Tillich M, Ranner G, Trummer M, Kleinert R. Symptomatic neuroepithelial (ependymal) cyst of the fourth ventricle: MR appearance. AJR Am J Roentgenol 1999;172:553-4.

12. Numaguchi Y, Foster RW, Gum GK. Large asymptomatic noncolloid neuroepithelial cysts in the lateral ventricle: CT and MR features. Neuroradiology 1989;31:98-101.

13. Periakaruppan A, Kesavadas C, Radhakrishnan VV, Thomas B,
Rao RM. Unique MR spectroscopic finding in colloid-like cyst. Neuroradiology 2008;50:137-44.

14. Majós C, Aguilera C, Cos M, Camins A, Candiota AP, Delgado-Goñi T, et al.In vivo proton magnetic resonance spectroscopy of intraventricular tumours of the brain. Eur Radiol 2009;19:2049-59.

15. Candiota AP, Majós C, Bassols A, Cabanas ME, Acebes JJ, Quintero MR, et al. Assignment of the $2.03 \mathrm{ppm}$ resonance in in vivo $1 \mathrm{H}$ MRS of human brain tumour cystic fluid: Contribution of macromolecules. MAGMA 2004;17:36-46

16. Lantos PL, Louis DN, Rosenblum MK, Kleihues P. Tumours of the nervous system. In: Graham DI, Lantos PL, editors. Greenfield's Neuropathology. $7^{\text {th }}$ ed., vol. 2. London: Arnold Publisher; 2002. p. 968-9.

Cite this article as: Hingwala DR, Radhakrishnan N, Kesavadas C, Thomas B, Kapilamoorthy TR, Radhakrishnan VV. Neuroenteric cysts of the braincomprehensive magnetic resonance imaging. Indian J Radiol Imaging 2013;23:155-63.

Source of Support: Nil, Conflict of Interest: None declared 